\title{
The Characteristic Feature of Thermal Convection in the Atmosphere (II) -Case of Moist Convection-
}

\author{
By K. Gambo and E. Machida \\ Electronic Computation Center, Japan Meteorological Agency \\ (Manuscript received 25 April 1968, in revised form 17 August 1968)
}

\begin{abstract}
Considering the moist convection from the standpoint of view of scale analysis of disturbance, we obtain the relation of conservation of kinetic and potential energy in a special case of shallow convection. From this energy conservation, the maximum vertical velocity of convective motion is estimated approximately as the function of the parameters such as the ratio of the cloud area to the associated cloudless area and the static stability of the large-scale enviromental atmosphere.

In order to examine the qualitative discussion mentioned above, the numerical experiment of thermal convection in a wet air case is performed. The results show a good agreement with those obtained by the qualitative discussion.
\end{abstract}

\section{Introduction}

In the previous paper (Gambo and Yoshida 1967) one of the authors treated the thermal convection in a special case of dry air. In that paper it was emphasized that the exponential amplification of amplitude of disturbance with time in dry convection was suppressed by the modification of mean static stability due to the upward eddy transport of sensible heat.

In the present paper the thermal convection in case of moist convection is examined. As pointed out by Ogura and Phillips (1962), the general treatment of moist convection is complicated one compared with that of dry convection, we consider at first the characteristic feature of moist convection from the standpoint of view of scale analysis of disturbance. By making use of the result obtained from the scale analysis, we express the vertical velocity of convective motion as the functions of the cloud amount, the mean static stability and so on.

If the vertical velocity of convective motion is estimated by the parameters such as the mean static stability and the cloud amount in the largescale motion, the contribution of convective motion to the large scale motion may be roughly obtained. Leith (1965) proposed the relation between the vertical velocity of convective motion and the mean static stability without the theoretical background and he tried to explain the vertical distribution of relative humidity in the tropical region. In this paper the theoretical consideration about the Leith's proposal mentioned above will be presented.

\section{The basic equations}

For the sake of simplicity, we consider the motion in a vertical two dimensional $(x, z)$ plane independent on $y$, where $(x, y, z)$ coordinates are the usual rectangular coordinates fixed on the earth.

The equations of motion in the $x$, and $z$-directions, the continuity equation and the thermodynamic equation may be written in the following way:

$$
\begin{gathered}
\frac{\partial u}{\partial t}+u \frac{\partial u}{\partial x}+w \frac{\partial u}{\partial z}=-\frac{1}{\rho} \frac{\partial p}{\partial x} \\
\frac{\partial w}{\partial t}+u \frac{\partial w}{\partial x}+w \frac{\partial w}{\partial z}+\rho g=-\frac{1}{\rho} \frac{\partial p}{\partial z} \\
\frac{\partial \rho}{\partial t}+\frac{\partial}{\partial x}(\rho u)+\frac{\partial}{\partial z}(\rho w)=0 \\
\frac{\partial \theta}{\partial t}+u \frac{\partial \theta}{\partial x}+w \frac{\partial \theta}{\partial z}=\frac{\theta}{c_{p} T} \frac{d Q}{d t}
\end{gathered}
$$

Here, the Coriolis force is neglected because we treat the small-scale convective motion and viscous and conduction terms are also neglected. The notations in (2.1)-(2.4) are $u=x$ component of 
velocity, $w=$ vertical component of velocity, $p=$ pressure, $\theta=$ potential temperature, $\rho=$ density, $g=$ gravitational acceleration, $c_{p}=$ specific heat at constant pressure, $T=$ temperature and $d Q / d t=$ rate of non-adiabatic heating per unit mass due to the release of latent heat of condensation of water vapor.

Now we consider the small disturbances superposed on a resting fundamental state of hydrostatic equilibrium. State variables $p, \rho, T$ and $\theta$ are expressed in the form

$$
\begin{gathered}
p(x, z, t)=p_{o}(z)+p^{\prime}(x, z, t) \\
\rho(x, z, t)=\rho_{o}(z)+\rho^{\prime}(x, z, t) \\
T(x, z: t)=T_{o}(z)+T^{\prime}(x, z, t) \\
\theta(x, z, t)=\theta_{o}(z)+\theta^{\prime}(x, z, t)
\end{gathered}
$$

where the suffix zero and the prime denote the fundamental and perturbation quantities respectively.

If we assume that all of $p^{\prime} / p_{o}, \rho^{\prime} / \rho_{o}, T^{\prime} / T_{o}$ and $\theta^{\prime} / \theta_{o}$ are comparable with or smaller than the magnitude of order $10^{-1}$, the following approximation may be allowed with good accuracy.

$$
\begin{gathered}
\ln p \doteqdot \ln p_{o}+p^{*} \\
\ln \rho \doteqdot \ln \rho_{o}+\rho^{*} \\
\ln T \doteqdot \ln T_{o}+T^{*} \\
\ln \theta \doteqdot \ln \theta_{o}+\theta^{*}
\end{gathered}
$$

where

$$
p^{*}=p^{\prime} / p_{o}, \rho^{*}=\rho^{\prime} / \rho_{o}, \theta^{*}=\theta^{\prime} / \theta_{o} \text { and } T^{*}=T^{\prime} / T_{o} .
$$

Replacing these relations in (2.1), (2.2), (2.3) and (2.4), we may easily obtain

$$
\begin{gathered}
\frac{\partial u}{\partial t}+u \frac{\partial u}{\partial x}+w \frac{\partial u}{\partial z}=-R T_{o} \frac{\partial p^{*}}{\partial x} \\
\frac{\partial w}{\partial t}+u \frac{\partial w}{\partial x}+w \frac{\partial w}{\partial z}=-R T_{o} \frac{\partial p^{*}}{\partial z}+g T^{*} \\
\frac{\partial \rho^{*}}{\partial t}+u \frac{\partial \rho^{*}}{\partial x}+w \frac{\partial \rho^{*}}{\partial z} \\
+\frac{1}{\rho_{o}}\left[\frac{\partial}{\partial x}\left(\rho_{o} u\right)+\frac{\partial}{\partial z}\left(\rho_{o} w\right)\right]=0 \\
\frac{\partial \theta^{*}}{\partial t}+u \frac{\partial \theta^{*}}{\partial x}+w \frac{\partial \theta^{*}}{\partial z}=-S w+\frac{1}{c_{p} T} \frac{d Q}{d t}
\end{gathered}
$$

where $S=(\partial / \partial z) \ln \theta_{o}$ and $R$ is the gas constant. Here we use the relation of hydrostatic equilibrium such as

$$
R T_{o} \frac{\partial}{\partial z} \ln p_{o}+g=0
$$

\section{Scale analysis of convective motion (I)}

The scale analysis of equations (2.5)-(2.8) was proposed by Ogura and Phillips by introducing the parameter $\varepsilon=\Delta \theta / \bar{\theta}$ where $\bar{\theta}$ is the mean value of potential temperature in the domain we concerned with and $\Delta \theta$ is the difference between maximum and minimum values of potential temperature. In this section, we use the same principle adopted by Ogura and Phillips. However the main purpose of our scale analysis is focused to find the appropriate equations of motion and the thermodynamic equation for the treatment of moist convection.

Now we introduce the characteristic length $D$ as the unit of length and the characteristic life time

$$
\tau^{2}=\left(g \frac{\partial}{\partial z} \ln \theta_{o}\right)^{-1}
$$

as the time unit. Then we have,

$$
\begin{gathered}
\bar{x}=D x^{\prime}, \quad z=D z^{\prime}, \quad t=\tau t^{\prime} \\
u=\frac{D}{\tau} u^{\prime}, \quad w=\frac{D}{\tau} w^{\prime}
\end{gathered}
$$

where the quantities with prime denote the nondimensional values with order of magnitude unity.

Here we assume the vertical scale of disturbance is of the same order with the horizontal one. It is well konwn that the use of Brunt-Vaisala's frequency as the characteristic life time of disturbance eliminates the acoustic wave.

By introducing non-dimensional variables such as $x^{\prime}, z^{\prime}, t^{\prime}, u^{\prime}$, and $w^{\prime}$ the equations (2.5) and (2.6) may be written as follows:

$$
\begin{aligned}
& \left(\frac{H_{m}}{\tau^{2} g}\right)\left(\frac{D}{H_{m}}\right)\left[\frac{\partial u^{\prime}}{\partial t^{\prime}}+\frac{\partial}{\partial x^{\prime}}\left(u^{\prime} u^{\prime}\right)+\frac{\partial}{\partial z^{\prime}}\left(u^{\prime} w^{\prime}\right)\right] \\
& =-\left(\frac{H_{m}}{D}\right) \frac{\partial p^{*}}{\partial x^{\prime}} \\
& \left(\frac{H_{m}}{\tau^{2} g}\right)\left(\frac{D}{H_{m}}\right)\left[\frac{\partial w^{\prime}}{\partial t^{\prime}}+\frac{\partial}{\partial x^{\prime}}\left(u^{\prime} w^{\prime}\right)+\frac{\partial}{\partial z^{\prime}}\left(w^{\prime} w^{\prime}\right)\right] \\
& =-\left(\frac{H_{m}}{D}\right) \frac{\partial p^{*}}{\partial z^{\prime}}+T^{*}
\end{aligned}
$$

where $H_{m}=R T_{o} / g$ and is regarded as the height of homogeneous atmosphere of temperature $T_{o}$.

From equations (3.3) and (3.4), the nondimensional quantities $p^{*}$ and $T^{*}$ may be expressed 
as follows

$$
\begin{aligned}
& p^{*} \sim \varepsilon\left(\frac{D}{H_{m}}\right)^{2} \\
& T^{*} \sim \varepsilon\left(\frac{D}{H_{m}}\right) \sim\left(\frac{H_{m}}{D}\right) p^{*}
\end{aligned}
$$

where the symbole " $\sim$ " means the same order of magnitude and $\varepsilon=H_{m} / \tau^{2} g$.

Since $\tau^{2}=\left(g \frac{\partial}{\partial z} \ln \theta_{o}\right)^{-1}$, we have

$$
\begin{gathered}
\tau^{2}=\left(g \frac{1}{\theta_{o}} \frac{\partial \theta_{o}}{\partial z}\right)^{-1} \sim\left(g \frac{1}{H_{m}} \cdot \frac{\Delta \theta_{o}}{\theta_{o}}\right)^{-1} \\
\varepsilon \sim \frac{\Delta \theta_{o}}{\theta_{o}}
\end{gathered}
$$

where $\Delta \theta_{o}$ is the difference between maximum and minimum values of $\theta_{0}$.

In case of the standard atmosphere, we may assume that

$$
\varepsilon \lesssim \frac{1}{10}
$$

Now we define the shallow convection as the case where $D / H_{m} \sim 1 / 10$ and the deep convection as $D / H_{m} \sim 1$. Concerning the potential temperature, we have from the relation of $\ln \theta=\ln T$ $x \ln p+$ const.

$$
\theta^{*}=T^{*}-\kappa p^{*}
$$

where $\kappa=R / c_{p} \approx 0.286$ and $\ln \theta_{o}=\ln T_{o}-\kappa \ln p_{o}$. In case of shallow convection, we may have the approximate relation between $\theta^{*}$ and $T^{*}$ from (3.6) and (3.8) in the following way

$$
\theta^{*}=T^{*}\left(1+O\left(D / H_{m}\right)\right) \approx T^{*}
$$

The value of $\theta^{*}$ is thus approximated by $T^{*}$ ignoring the effect of the dynamic pressure on $T^{*}$. This conclusion in case of shallow convection will be used frequently in the following sections. The value of $\rho^{*}$ is also approximated as follows:

$$
T^{*} \sim \rho^{*} \sim \varepsilon\left(D / H_{m}\right)
$$

because $\rho^{*}=p^{*}-T^{*}=-T^{*}\left(1+O\left(D / H_{m}\right)\right)$.

\section{Scale analysis of convective motion (II)}

In the foregoing section, we obtain the relation between $\theta^{*}, T^{*}, \rho^{*}$ and $p^{*}$. In this section, we consider the problem concerning the kinetic and potential energy. Before we discuss such a problem, we examine the continuity equation from the point of view of scale analysis. If we use the non-dimensional variables defined in (3.1) and (3.2), the continuity equation (2.3) becomes as follows:

$$
\begin{gathered}
\frac{1}{\tau} \frac{\partial}{\partial t^{\prime}} \ln \rho+\frac{1}{\tau}\left[\frac{1}{\rho} \frac{\partial}{\partial x^{\prime}}\left(\rho u^{\prime}\right)+\frac{\partial w^{\prime}}{\partial z^{\prime}}\right] \\
+\frac{D}{\tau} w^{\prime}\left(\frac{\partial}{\partial z} \ln \rho\right)=0 .
\end{gathered}
$$

By replacing $\ln \rho_{o}=\ln \rho_{0}+\rho^{*}$ in (4.1), we have

$$
\frac{\partial \rho^{*}}{\partial t^{\prime}}+\left(\frac{\partial u^{\prime}}{\partial x^{\prime}}+\frac{\partial w^{\prime}}{\partial z^{\prime}}\right)+D w^{\prime}\left(\frac{\partial}{\partial z} \ln \rho_{o}\right)=0 .
$$

Here we assume that $\rho_{o}$ is a function of $z$ for the sake of simplicity.

If we define the characteristic value of $\rho^{*}$ as $\tilde{\rho}^{*}$, we may put

$$
\rho^{*}=\tilde{\rho}^{*} \rho^{* \prime}
$$

where $\rho^{* /}$ is the non-dimensional value with order of magnitude unity. Thus the equation (4.2) may be written in the following way:

$$
\begin{aligned}
\tilde{\rho}^{*}\left(\frac{\partial \rho^{* \prime}}{\partial t^{\prime}}\right) & +\left(\frac{\partial u^{\prime}}{\partial x^{\prime}}+\frac{\partial w^{\prime}}{\partial z^{\prime}}\right) \\
& +D w^{\prime}\left(\frac{\partial}{\partial z} \ln \theta_{o}+\frac{g}{c^{2}}\right)=0
\end{aligned}
$$

where $c^{2}=\frac{c_{p}}{c_{v}} R T_{o}\left(c_{v}:\right.$ specific heat at constant volume). Here we use

$$
\frac{\partial}{\partial z} \ln \rho_{o}=\frac{\partial}{\partial z} \ln \theta_{o}+\frac{c_{v}}{c_{p}}\left(\frac{g}{R T_{o}}\right) .
$$

Since

$$
\frac{\partial}{\partial z} \ln \theta_{o} \sim \frac{1}{H_{m}} \cdot \frac{\Delta \theta_{o}}{\theta_{o}}=\frac{\varepsilon}{\dot{H}_{m}}
$$

and

$$
\frac{g}{c^{2}} \sim \frac{g}{R T_{o}}=\frac{1}{H_{m}},
$$

we may write approximately the equation (4.3) as follows:

$$
\begin{aligned}
\tilde{\rho}^{*}\left(\frac{\partial \rho^{* \prime}}{\partial t^{\prime}}\right) & +\left(\frac{\partial u^{\prime}}{\partial x^{\prime}}+\frac{\partial w^{\prime}}{\partial z^{\prime}}\right)+\frac{D}{H_{m}} \\
& \times\left[w^{\prime}\left(\frac{c_{v}}{c_{p}}+O(\varepsilon)\right)\right]=0
\end{aligned}
$$


The quantities designated by the parenthesis have where the order of magnitude unity. As shown already in section 3 , the order of magnitude of $\rho^{*}$ or $\tilde{\rho}^{*}$ is given by (3.10), i. e.,

$$
\rho^{*} \sim \tilde{\rho}^{*} \sim \varepsilon\left(D / H_{m}\right)
$$

Therefore the continuity equation (4.4) or (4.3) is approximated in the following way:

(a) case of shallow convection $\left(D / H_{m} \sim 1 / 10\right)$ :

$$
\frac{\partial u^{\prime}}{\partial x^{\prime}}+\frac{\partial w^{\prime}}{\partial z^{\prime}}=0 \quad \text { or } \quad \frac{\partial u}{\partial x}+\frac{\partial w}{\partial z}=0
$$

(b) case of deep convection $\left(D / H_{m} \sim 1\right)$ :

$$
\begin{gathered}
\frac{\partial u^{\prime}}{\partial x^{\prime}}+\frac{\partial w^{\prime}}{\partial z^{\prime}}-D w^{\prime}\left(\frac{\partial}{\partial z} \ln \theta_{o}+\frac{g}{C^{2}}\right)=0 \\
\text { or } \frac{\partial}{\partial x}\left(\rho_{o} u\right)+\frac{\partial}{\partial z}\left(\rho_{o} w\right)=0 .
\end{gathered}
$$

These results were also pointed out by Ogura and Phillips by the different method from our treatment. The approximate equation of continuity, (4.5) or (4.6) are used for the discussion of energy consideration.

Now we consider the rate of change of kinetic energy. The fundamental equations are given from (2.5) and (2.6) in the form:

$$
\begin{gathered}
\frac{\partial u}{\partial t}+u \frac{\partial u}{\partial x}+w \frac{\partial u}{\partial z}=-R T_{o} \frac{\partial p^{*}}{\partial x} \\
\frac{\partial w}{\partial t}+u \frac{\partial w}{\partial x}+w \frac{\partial w}{\partial z}=-R T_{o} \frac{\partial p^{*}}{\partial z}+g T^{*} .
\end{gathered}
$$

By multiplying $\rho_{o} u$ to (4.7) and $\rho_{o} w$ to (4.8) respectively, we get

$$
\begin{aligned}
\frac{d}{d t}\left(\rho_{o} K\right) \equiv & \frac{\partial}{\partial t}\left(\rho_{o} K\right)+\frac{\partial}{\partial x}\left(\rho_{o} u K\right)+\frac{\partial}{\partial z}\left(\rho_{o} w K\right) \\
= & -R T_{o}\left(\rho_{o} u \frac{\partial p^{*}}{\partial x}+\rho_{o} w \frac{\partial p^{*}}{\partial z}\right) \\
& +\rho_{o} g w T^{*} .
\end{aligned}
$$

Here we have used the continuity equation (4.6). Rearrangement of right-hand side of (4.9) gives the following relation

$$
\begin{aligned}
\frac{d}{d t}\left(\rho_{o} K\right)= & -R\left[\frac{\partial}{\partial x}\left(\rho_{o} u T_{o} p^{*}\right)+\frac{\partial}{\partial z}\left(\rho_{o} w T_{o} p^{*}\right)\right] \\
& +R p^{*} \rho_{o} w \frac{\partial T_{o}}{\partial z}+\rho_{o} g w T^{*}
\end{aligned}
$$

$$
K=\frac{1}{2}\left(u^{2}+w^{2}\right)
$$

Thus the integration over the domain we concerned with becomes

$$
\begin{aligned}
\frac{\partial}{\partial t} \iint \rho_{o} K d x d z=\int & \int\left(R p^{*} \rho_{o} w \frac{\partial T_{o}}{\partial z}+\rho_{o} g w T^{*}\right) \\
& \times d x d z .
\end{aligned}
$$

In deriving (4.11) from (4.10), it is assumed that the normal components of velocity vanish at boundaries or that the motion is periodic in the $x$-direction. The interesting point in (4.11) is that the first term of the right-hand side is of the order of $\varepsilon\left(D / H_{m}\right)$ compared with the second term. The order of magnitude of $R p^{*} \rho_{o} w \frac{\partial T_{o}}{\partial z}$ in (4.11) may be written in the form:

$$
\begin{aligned}
R p^{*} \rho_{o} w \frac{\partial T_{o}}{\partial z} \sim & R p^{*} \rho_{o} w \frac{\Delta \theta_{o}}{H_{m}}=\left(\frac{R T_{o}}{g}\right)\left(\frac{\theta_{o}}{T_{o}}\right) \\
& \times\left(\frac{\varepsilon}{H_{m}}\right)\left(\rho_{o} g w p^{*}\right)=\left(\frac{\theta_{o}}{T_{o}}\right) \\
& \times \varepsilon\left(\rho_{o} g w p^{*}\right) \sim \varepsilon \rho_{o} g w p^{*} .
\end{aligned}
$$

By making use of $p^{*} \sim \frac{D}{H_{m}} T^{*}$, we have

$$
R p^{*} \rho_{o} w \frac{\partial T_{o}}{\partial z} \sim \varepsilon\left(\frac{D}{H_{m}}\right)\left(\rho_{o} g w T^{*}\right) .
$$

Therefore in case of shallow convection we may have the following equation with sufficient accuracy

$$
\frac{\partial}{\partial t} \iint \rho_{o} K d x d z=\iint \rho_{o} g w T^{*} d x d z .
$$

If we replace simply $T^{*}=\theta^{*}+\kappa p^{*}$ in (4.11), we have

$$
\begin{aligned}
& \frac{\partial}{\partial t} \iint \rho_{o} K d x d z=\iint \rho_{o} g w \theta^{*} d x d z \\
& \quad+\iint \rho_{o} g w p^{*}\left(\frac{R T_{o}}{g}\right) \frac{\partial}{\partial z} \ln \theta_{o} d x d z
\end{aligned}
$$

where

$$
\frac{\partial}{\partial z} \ln \theta_{o}=\frac{1}{T_{o}}\left(\frac{\partial T_{o}}{\partial z}+\frac{g}{c_{p}}\right) .
$$


Since $\frac{R T_{o}}{g} \frac{\partial}{\partial z} \ln \theta_{o} \sim \varepsilon$ and $p^{*} \sim \frac{D}{H_{m}} \theta^{*}$, the equation (4.14) may be written

$$
\begin{aligned}
\frac{\partial}{\partial t} \iint \rho_{o} K d x d z & =\iint \rho_{o} g w \theta^{*}\left[1+O\left(\varepsilon \frac{D}{H_{m}}\right)\right] d x d z \\
& \approx \iint \rho_{o} g w \theta^{*} d x d z
\end{aligned}
$$

If we assume that the basic state of atmosphere is such as $\partial \theta_{o} / \partial z=0$, the relaion (4.15) holds exactly. However, it must be emphasized that the relation (4.15) may be satisfied even if $\partial \theta_{o} / \partial z$ $\neq 0$ with sufficient accuracy provided that $\varepsilon \sim 1 / 10$. From this consideration we may understand that the important factor for selecting the basic state of atmosphere is the magnitude of $\varepsilon$.

\section{The rate of change of potential temperature}

In case of dry convection, the thermodynamic equation (2.8) is written as follows:

$$
\frac{\partial \theta^{*}}{\partial t}+u \frac{\partial \theta^{*}}{\partial x}+w \frac{\partial \theta^{*}}{\partial z}=-S w
$$

where

$$
S=\frac{\partial}{\partial z} \ln \theta_{o}=\frac{1}{T_{o}}\left(\frac{\partial T_{o}}{\partial z}+\Gamma\right) .
$$

Thus the rate of change of potential energy $P$ may be written by multiplying $g z$ on both sides of (5.1) and making use of continuity equation (4.6) as follows:

$$
\begin{gathered}
\frac{\partial P}{\partial t}+u \frac{\partial P}{\partial x}+w \frac{\partial P}{\partial z}=-g w \theta^{*}+g S w z \\
\text { or } \frac{\partial}{\partial t}\left(\rho_{o} P\right)+\frac{\partial}{\partial x}\left(\rho_{o} u P\right)+\frac{\partial}{\partial z}\left(\rho_{o} w P\right) \\
=-\rho_{o} g w \theta^{*}+\rho_{o} g S w z
\end{gathered}
$$

where

$$
P=-g \theta^{*} z \text {. }
$$

If we assume the cyclic boundary condition in the $x$-direction, we have $\int S w d x=0$. Therefore we have the energy equation from (4.15) and (5.3)

$$
\frac{\partial}{\partial t} \iint \rho_{o}(K+P) d x d z=0 .
$$

Here we assume that the normal components of velocity vanish at the top and bottom boundaries. In case of shallow convection we may be able to put $\theta^{*}=T^{*}$ with sufficient accuracy as mentioned in section 3. Therefore we get

$$
\frac{\partial}{\partial t} \iint \rho_{o}\left(K-g T^{*} z\right) d x d z=0 .
$$

In case of moist convecction, however, the energy equation becomes complicated one (Ogura and Phillips). The energy equation is not expected to be simple as well as the equation (5.3). Considering the difficult situation to derive the energy equation in case of moist convection, we consider to obtaine the relation between the kinetic and potential energy of moist converction under the appropriate assumptions. For the sake of simplicity, the shallow convection is assumed in the following discussion.

Now we assume that the convective motion is schematically expressed as shown in Fig. 1. The domain of upward current is occupied by the length of $\sigma$ on the horizontal plane, while the downward current exists in the length of $(1-\sigma)$. In this case, the thermodynamic equation becomes as follows:

$$
\begin{aligned}
& \frac{\partial T_{e}}{\partial t}+u_{e} \frac{\partial T_{e}}{\partial x}+w_{e}\left(\frac{\partial T_{e}}{\partial z}+\Gamma\right)=0 \\
& \frac{\partial T_{c}}{\partial t}+u_{c} \frac{\partial T_{c}}{\partial x}+w_{c}\left(\frac{\partial T_{c}}{\partial z}+\Gamma_{e}\right)=0
\end{aligned}
$$

where the suffix $c$ or $e$ denotes the value of upward or downward domain, $\Gamma_{e}$ is the moist adiabatic lapse rate and

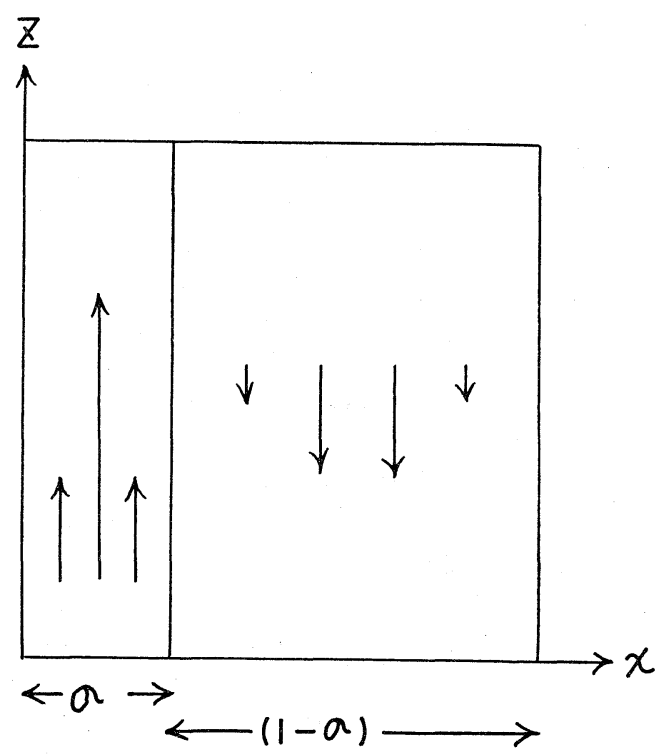

Fig. 1. The area of cumulus clouds. 


$$
\Gamma_{e}=\Gamma+\frac{L}{c_{p}} F^{*} .
$$

Here we assume that the air is saturated in the domain of upward current and

$$
\frac{d Q}{d t}=-\frac{L}{c_{p}} \frac{d q_{s}}{d t}=-\frac{L}{c_{p}} w F^{*}
$$

( $q_{s}$ : saturation specific humidity, $L$ : latent heat of condensation). $\quad F^{*}$ is the function of temperature and pressure. The relation between $\Gamma_{e}, \Gamma, F^{*}$ and $q_{s}$ is shown in the Appendix.

The continuity equation (4.5) of shallow convection is written in our case

$$
\begin{aligned}
& \frac{\partial u_{e}}{\partial x}+\frac{\partial w_{e}}{\partial z}=0 \\
& \frac{\partial u_{c}}{\partial x}+\frac{\partial w_{c}}{\partial z}=0 .
\end{aligned}
$$

Combining the equations (5.6) and (5.7) with the equations (5.4) and (5.5), we get

$$
\begin{gathered}
\frac{\partial T_{e}}{\partial t}+\frac{\partial}{\partial x}\left(u_{e} T_{e}\right)+\frac{\partial}{\partial z}\left(w_{e} T_{e}\right)+w_{e} \Gamma=0 \\
\frac{\partial T_{c}}{\partial t}+\frac{\partial}{\partial x}\left(u_{c} T_{c}\right)+\frac{\partial}{\partial z}\left(w_{c} T_{c}\right)+w_{c} \Gamma \\
=w_{c}\left(\frac{L}{c_{p}}\right) F^{*} .
\end{gathered}
$$

Under the assumption of cyclic or solid boundary condition, we have

$$
\frac{\partial}{\partial t}\langle\bar{T}\rangle=\frac{L}{c_{p}}\left\langle\overline{\left.w_{c} F^{*}\right\rangle}\right\rangle=\frac{L}{c_{p}} \int_{\sigma}\left\langle w_{c} F^{*}\right\rangle d x .
$$

Here the symbols "__ and " $>>$ " denote the mean values in the horizontal and vertical directions respectively and $\int_{\sigma} d x$ means the integration with respect to $x$ within the upward current. In deriving the equation (5.10), the assumption that there is no heat exchange through the boundary between the upward and downward currents is also used.

The equation (5.10) menas the uniform heating of the domain we concerned with due to the release of latent heat of condensation. Taking into account of this uniform heating, we consider to simplify the equations (5.8) and (5.9). In the following discussion, we assume, for the sake of simplicity that

$$
\Gamma_{e}=\Gamma-\frac{L}{c_{p}} F^{*}
$$

is constant with height and the convective motion to be considered is bounded at the bottom by the earth surface, $z=0$ and at the top by the level, $z=z_{M}$. (see Fig. 2). If we define $T_{e}{ }^{\prime}, T_{c}{ }^{\prime}$, $T_{M e}$ and $T_{M c}$ in the following way,

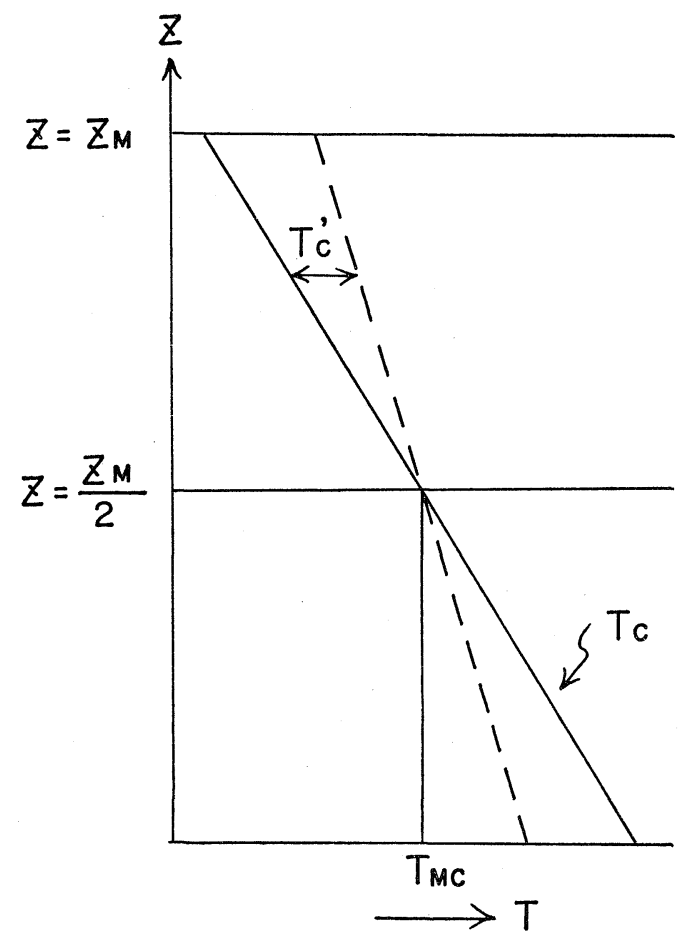

Fig. 2. The vertical distribution of temperature. The dashed line is moist adiabat.

$$
\begin{gathered}
T_{e}=T_{M e}+\Gamma\left(\frac{z_{M}}{2}-z\right)+T_{e^{\prime}} \\
\frac{\partial T_{M e}}{\partial t}=0 \\
T_{c}=T_{M c}+\Gamma_{e}\left(\frac{z_{M}}{2}-z\right)+T_{c}^{\prime} \\
\frac{\partial T_{M c}}{\partial t}=\frac{\partial}{\partial t}\langle\bar{T}\rangle,
\end{gathered}
$$

the requirement for $T_{e}^{\prime}$ and $T_{c}^{\prime}$ becomes as follows

$$
\frac{\partial}{\partial t}\left[\int_{1-\sigma}\left(\int T_{e^{\prime}} d z\right) d x+\int_{\sigma}\left(\int T_{c^{\prime}} d z\right) d x\right]=0
$$


where $\int_{1-\sigma} d x$ and $\int_{\sigma} d x$ denote the integration with respect to $x$ within the downward and upward currents respectively. The relations between $T_{e}, T_{e}^{\prime}$, and $T_{M e}$ or $T_{c}, T_{c}^{\prime}$ and $T_{M c}$ are shown in Fig. 2 schematically. $T_{M e}$ and $T_{M c}$ denote the values of $T_{e}$ and $T_{c}$ at the level $z=$ $z_{M} / 2$. Thus we have

$$
\begin{gathered}
T_{e}=T_{o e}+T_{e}{ }^{\prime} \\
T_{c}=T_{o c}+T_{c}{ }^{\prime}
\end{gathered}
$$

where

$$
\begin{aligned}
T_{o e} & =T_{M e}+\Gamma\left(\frac{z_{M}}{2}-z\right) \\
\text { and } T_{o c} & =T_{M c}+\Gamma_{e}\left(\frac{z_{M}}{2}-z\right) .
\end{aligned}
$$

The values of $T_{o e}$ and $T_{o c}$ may be regarded as those of resting fundamental state of hydrostatic equilibrium in the downward and upward currents respectively. The equations for $T_{e}{ }^{\prime}$ and $T_{c}{ }^{\prime}$ may be written in the form

$$
\begin{aligned}
& \frac{\partial T_{e}^{\prime}}{\partial t}+\frac{\partial}{\partial x}\left(u_{e} T_{e}^{\prime}\right)+\frac{\partial}{\partial z}\left(w_{e} T_{e}^{\prime}\right)=0 \\
& \frac{\partial T_{c}^{\prime}}{\partial t}+\frac{\partial}{\partial x}\left(u_{c} T_{c}^{\prime}\right)+\frac{\partial}{\partial z}\left(w_{c} T_{c}^{\prime}\right)=0 .
\end{aligned}
$$

Rearrangements of (5.13) and (5.14) through the continuity equation become as follows:

$$
\begin{aligned}
& \frac{\partial}{\partial t}\left(\rho_{o} T_{e}{ }^{\prime} z\right)+\frac{\partial}{\partial x}\left(\rho_{o} u_{e} T_{e}{ }^{\prime} z\right)+\frac{\partial}{\partial z}\left(\rho_{o} w_{e} T_{e}{ }^{\prime} z\right) \\
& \quad=\rho_{o} w_{e} T_{e}^{\prime} \\
& \frac{\partial}{\partial t}\left(\rho_{o} T_{c}^{\prime} z\right)+\frac{\partial}{\partial x}\left(\rho_{o} u_{c} T_{c}^{\prime} z\right)+\frac{\partial}{\partial z}\left(\rho_{o} w_{c} T_{c}{ }^{\prime} z\right) \\
& \quad=\rho_{o} w_{c} T_{c}{ }^{\prime} .
\end{aligned}
$$

In order to obtain the rate of change of potential energy, we divide (5.15) by $T_{o c}$ and (5.16) by $T_{o c}$ respectively and we get

$$
\begin{gathered}
\frac{\partial}{\partial t}\left(\rho_{o} T_{e}^{*} z\right)+\frac{\partial}{\partial x}\left(\rho_{o} u_{e} T_{e}^{*} z\right)+\frac{\partial}{\partial z}\left(\rho_{o} w_{e} T_{e}^{*} z\right) \\
=\rho_{o} w_{e} T_{e}^{*}-\left(\rho_{o} w_{e} T_{e} z^{*} \frac{1}{T_{o e}} \frac{\partial T_{o e}}{\partial z}\right) \\
\frac{\partial}{\partial t}\left(\rho_{o} T_{c}^{*} z\right)+\frac{\partial}{\partial x}\left(\rho_{o} u_{c} T_{c}^{*} z\right)+\frac{\partial}{\partial z}\left(\rho_{o} w_{c} T_{c}^{*} z\right) \\
=\rho_{o} w_{c} T_{c}^{*}-\left(\rho_{o} w_{c} T_{c}{ }^{*} z \frac{1}{T_{o c}} \frac{\partial T_{o c}}{\partial z}\right.
\end{gathered}
$$

$$
\left.+\rho_{o} T_{c}^{*} z \frac{1}{T_{o c}} \frac{\partial T_{o c}}{\partial t}\right)
$$

where

$$
T_{e}^{*}=\frac{T_{e}^{\prime}}{T_{o e}} \quad \text { and } \quad T_{c}^{*}=\frac{T_{c}^{\prime}}{T_{o c}} .
$$

In case of shallow convection, we may put, introducing $\varepsilon$ and $\tau$ mentioned in section 3 , as follows:

$$
\begin{aligned}
& \frac{1}{T_{o \epsilon}}\left|\frac{\partial T_{o e}}{\partial z}\right| \lesssim \varepsilon \frac{1}{z_{M}}, \quad \frac{1}{T_{o c}}\left|\frac{\partial T_{o c}}{\partial z}\right| \lesssim \varepsilon \frac{1}{z_{M}}, \\
& \frac{1}{T_{o c}}\left|\frac{\partial T_{o c}}{\partial t}\right| \lesssim \varepsilon \frac{1}{\tau} .
\end{aligned}
$$

In this sense, the second terms of the right-hand side of (5.17) and (5.18) may be neglected compared with other terms. Therefore we have with sufficient accuracy

$$
\begin{aligned}
& \frac{\partial}{\partial t}\left(\rho_{o} T_{e}^{*} z\right)+\frac{\partial}{\partial x}\left(\rho_{o} u_{e} T_{e}^{*} z\right)+\frac{\partial}{\partial z}\left(\rho_{o} w_{e} T_{e}^{*} z\right) \\
& =\rho_{o} w_{e} T_{e}^{*} \\
& \frac{\partial}{\partial t}\left(\rho_{o} T_{c}^{*} z\right)+\frac{\partial}{\partial x}\left(\rho_{o} u_{c} T_{c}^{*} z\right)+\frac{\partial}{\partial z}\left(\rho_{o} w_{c} T_{c}^{*} z\right) \\
& =\rho_{o} w_{c} T_{c}^{*} .
\end{aligned}
$$

On the other hand, the rate of change of kinetic energy in case of shallow convection may be easily obtained from (4.10) with sufficient accuracy as follows:

$$
\begin{aligned}
& \frac{\partial}{\partial t}\left(\rho_{o} K_{e}\right)+\frac{\partial}{\partial x}\left(\rho_{o} u_{e} K_{e}\right)+\frac{\partial}{\partial z}\left(\rho_{o} w_{e} K_{e}\right) \\
&= \rho_{o} g w_{e} T_{e}^{*}-R\left[\frac{\partial}{\partial x}\left(\rho_{o} u_{e} T_{o e} p_{e}^{*}\right)\right. \\
&\left.+\frac{\partial}{\partial z}\left(\rho_{o} w_{e} T_{o e} p_{e}^{*}\right)\right] \\
& \frac{\partial}{\partial t}\left(\rho_{o} K_{c}\right)+\frac{\partial}{\partial x}\left(\rho_{o} u_{c} K_{c}\right)+\frac{\partial}{\partial z}\left(\rho_{o} w_{c} K_{c}\right) \\
&=\rho_{o} g w_{c} T_{c}^{*}-R\left[\frac{\partial}{\partial x}\left(\rho_{o} u_{c} T_{o c} p_{c}^{*}\right)\right. \\
&\left.\quad+\frac{\partial}{\partial z}\left(\rho_{o} w_{c} T_{o c} p_{c}^{*}\right)\right]
\end{aligned}
$$

where

$$
p_{e}^{*}=\frac{p_{e}^{\prime}}{p_{e o}} \quad \text { and } \quad p_{c}^{*}=\frac{p_{c}^{\prime}}{p_{c o}}
$$

( $p_{e o}$; pressure corresponding to $T_{o e}, p_{c o}$ : pressure corresponding to $T_{o c}$ ). 
Assuming the cyclic or solid boundary condition, motion.

the energy integral may be given by

$$
\begin{aligned}
\frac{\partial}{\partial t} & {\left[\iint_{1-\sigma} \rho_{o} K_{e} d x d z+\iint_{\sigma} \rho_{o} K_{c} d x d z\right.} \\
& \left.-\iint_{1-\sigma} \rho_{o} g z T_{e}^{*} d x d z-\iint_{\sigma} \rho_{o} g z T_{c}^{*} d x d z\right] \\
& =0 .
\end{aligned}
$$

In deriving (5.23) from (5.19)-(5.22), we assmue, for the sake of simplicity, that there is no potential and kinetic energy exchange through the boundary of the upward and downward currents. In case of deep convection, the authors could not find the simple relation such as (5.23).

\section{Estimation of maximum kinetic energy}

As mentioned in the previous section, we have the energy integral of convective motion. From the energy integral, we may expect that the decrease of potential energy corresponds to the increase of kinetic energy. In case of dry convection, the circumstance mentioned above will be clearly explained. The energy integral (5.3) for dry convection is written in the form,

where

$$
\left\langle\rho_{o}(\bar{K}+\bar{P})\right\rangle=\text { const. }
$$

$$
P=-g z \theta^{*} \quad \text { and } \quad K=\frac{1}{2}\left(u^{2}+w^{2}\right)
$$

In case of shallow convection, the potential energy $P$ is approximated by $P=-g z T^{*}$. The conservation of total energy of dry convection gives the following relation

$$
\left\langle\rho_{o}(\bar{K}+\bar{P})\right\rangle_{t=0}=\left\langle\rho_{o}(\bar{K}+\bar{P})\right\rangle_{t=\tau}
$$

where the suffix $t=0$ or $t=\tau$ denotes the value at $t=0$ or $t=\tau$. If we select the initial conditions for $K$ and $P$ as follows

$$
\langle\bar{K}\rangle_{t=0}=0, \quad\langle\bar{P}\rangle_{t=0} \equiv\langle\bar{P}\rangle_{0},
$$

the maximum value of $\langle\bar{K}\rangle$ will be obtained when the value of $\langle\bar{P}\rangle$ becomes minimum, i. e., $\langle\bar{P}\rangle=0$. Here $\rho_{o}$ is regarded as constant because we are concerned with the shallow convection. Thus we have

$$
\langle\bar{K}\rangle_{\max }=\langle\bar{P}\rangle_{t=0}
$$

Here we assume that $\langle\bar{P}\rangle$ is always positive because we are considering the unstable convective
Now we consider to express $\langle\bar{P}\rangle$ as the function of $\theta_{0}$ and $\theta^{\prime}$. From the definition of $\theta^{*}$, we have

$$
\theta=\theta_{o}+\theta^{\prime}, \quad \ln \theta=\ln \theta_{o}+\theta^{*}, \quad \theta^{*}=\theta^{\prime} \mid \theta_{o} .
$$

If we choose $\theta_{o}$ such as $\partial \theta_{o} / \partial z=0$, the thermodynamic equation (5.1) becomes

$$
\frac{\partial \theta^{*}}{\partial t}+\frac{\partial}{\partial x}\left(u \theta^{*}\right)+\frac{\partial}{\partial z}\left(w \theta^{*}\right)=0
$$

This means that $\partial / \partial t\left\langle\bar{\theta}^{*}\right\rangle=0$. Thus, under the assumption that the convective motion is bounded in the vertical direction such as shown in Fig. 2, we may write $\theta^{*}$ in the following way:

$$
\begin{aligned}
& \theta^{*}=\frac{\theta^{\prime}}{\theta_{o}} \div-\left[\frac{1}{\theta_{0}}\left(\frac{\partial \theta^{\prime}}{\partial z}\right)\right]_{z M / 2} \cdot\left(z_{M / 2}-z\right), \\
& \left\langle\theta^{*}\right\rangle=0
\end{aligned}
$$

where the suffix $z_{M} / 2$ denotes the value at the level $z=z_{M} / 2$. In the equation (6.5), $\theta^{*}$ is approximated by the linear function of $z$, assuming that the stability factor

$$
\frac{\partial \theta}{\partial z}\left(=\frac{\partial \theta_{o}}{\partial z}+\frac{\partial \theta^{\prime}}{\partial z}=\frac{\partial \theta^{\prime}}{\partial z}\right)
$$

is constant with height for the sake of simplicity. In this case, the potential energy at the initial time becomes

$$
\begin{aligned}
\langle\vec{P}\rangle_{t=0} & =g \bar{S}_{t=0} \cdot \frac{\left\langle\left(\frac{z_{M}}{2}-z\right) z\right\rangle}{z_{M}} \\
& =-\frac{g}{12} z_{M}{ }^{2} \bar{S}_{t=0}
\end{aligned}
$$

where

$$
\bar{S}=\left(\frac{1}{\theta_{o}} \frac{\partial \bar{\theta}}{\partial z}\right)_{z=z_{M} / 2} .
$$

Then, the maximum value of kinetic energy $\langle\bar{K}\rangle_{\max }$ may be approximated from (6.3) and (6.6) as follows:

$$
\langle\bar{K}\rangle_{\max }=-\frac{g}{12} z_{M}^{2}\langle\bar{S}\rangle_{t=0} .
$$

If we use $P=-g z T^{*}$ instead of $P=-g z \theta^{*}$ as a case of shallow convection, the relations corresponding to (6.5)-(6.7) may be written 


$$
\begin{aligned}
T^{*}= & \frac{T^{\prime}}{T_{o}}=-\frac{1}{T_{o}}\left(\frac{\partial T}{\partial z}+\Gamma\right)_{z=z_{M} / 2} \\
& \times\left(\frac{z_{M}}{2}-z\right) \\
\langle\bar{P}\rangle_{t=0}= & -\frac{1}{T_{o}}\left(\frac{\partial \bar{T}}{\partial z}+\Gamma\right)_{z=z_{M} / 2} \cdot \frac{g z_{M}{ }^{2}}{12} \\
\langle\bar{K}\rangle_{\max }= & -\frac{g z_{M}{ }^{2}}{12}\left[\frac{1}{T_{o}}\left(\frac{\partial \bar{T}}{\partial z}+\Gamma\right)\right]_{z=z_{M} / 2, t=0 .}
\end{aligned}
$$

By making use of (6.7) or (6.10), we try to estimate the maximum value of kinetic energy in the following section.

Here we would like to add some comment about the relation of (6.2). In our previous paper (Gambo and Yoshida), we derived the following relation, introducing the characteristic wave number $m$ in the vertical direction,

$$
\langle\bar{K}\rangle-\frac{g}{m^{2}}\langle\bar{S}\rangle=\text { const. }
$$

The relation of (6.7) or (6.10) gives the value of $m$ as follows:

$$
\frac{1}{m^{2}}=\frac{1}{12} z_{M^{2}}=\left(\frac{z_{M}}{\pi}\right)^{2} \cdot\left(\frac{\pi}{12}\right)^{2} \div\left(\frac{z_{M}}{\pi}\right)^{2} \times 0.82 .
$$

In the common sense of definition of charact eristic wave number, $m^{2}$ may be regarded as $\frac{1}{m^{2}} \approx\left(\frac{z_{M}}{\pi}\right)^{2}$. In this sense, we may conclude that the rough estimation for the energy integral about $\langle\bar{K}\rangle$ and $\langle\bar{P}\rangle$ which was derived in our previous paper was reasonable one.

\section{Numerical experiment in dry air case}

In order to check our results which we derived theoretically until the preceeding sections, we perform the numerical experiment in case of shallow convection of dry air.

In our case, the equations of motion (2.5) and (2.6) are rewritten by making use of continuity equation (4.5) in the form of vorticity equation

$$
\frac{\partial \zeta}{\partial t}=J(\psi, \zeta)+g \frac{\partial T^{*}}{\partial z}
$$

where

$$
\zeta=\frac{\partial u}{\partial z}-\frac{\partial w}{\partial x}, \quad u=\frac{\partial \psi}{\partial z} \text { and } w=-\frac{\partial \psi}{\partial x} .
$$

Here $\phi$ is the stream function and $\mathbf{J}$ is the Jacobian operator such as

$$
\mathbf{J}(\psi, \zeta)=\frac{\partial \psi}{\partial x} \frac{\partial \zeta}{\partial z}-\frac{\partial \psi}{\partial z} \frac{\partial \zeta}{\partial x} .
$$

The thermodynamic equation corresponding to (5.1) may be written in case of shallow convection

$$
\frac{\partial T^{*}}{\partial t}=\mathbf{J}\left(\phi, T^{*}\right)+\bar{S} \frac{\partial \psi}{\partial x}
$$

where

$$
\bar{S}=\frac{1}{T_{o}}\left(\frac{\partial \bar{T}}{\partial z}+\Gamma\right) .
$$

In the numerical experiment, $\bar{S}$ is regarded as

$$
\bar{S}=\frac{1}{\left\langle T_{0}\right\rangle}\left(\frac{\partial \bar{T}}{\partial z}+\Gamma\right) .
$$

The finite-difference coordinates for the numerical computation will be $i$ and $j$, and

$$
\begin{array}{ll}
x=i \Delta x, & i=1,2, \cdots \cdots I \\
z=j \Delta z, & j=1,2, \cdots \cdots J
\end{array}
$$

where $\Delta x$ and $\Delta z$ are grid-sizes in the horizontal and vertical directions respectively.

Here the bottom and top boundary surfaces are specified by $j=1$ and $j=J$ respectively and the side boundary surfaces are specified by $i=1$ and $i=I$.

In the actual computation, we assume that $\Delta x=\Delta z=d$, and the upper and lower boundaries are considered to be rigid surfaces. It is further assumed that variables such as the stream function $\phi$ and $T^{*}$ at $i=i$ are the same as those at $i=I$ $+i$. The finite difference schemes for the equations (7.1) and (7.2) are not reproduced here, because the details are written in our previous paper. (Gambo and Yoshida).

In the numerical computation, we use the following constants:

$$
d=500 \mathrm{~m}, \quad I=200, \quad J=11 \text { and } \Delta t=10 \mathrm{sec} .
$$

where $\Delta t$ is the time increment which we use for the time integration. The initial conditions for $u, w$ and $T^{\prime}$ are as follows:

$$
u=0, \quad w=0,
$$

$$
T^{\prime}=A \sin \frac{2 \pi}{L} x \sin \frac{2 \pi}{D} z \quad \text { when } z<2.5 \mathrm{~km} \text {, }
$$$$
T^{\prime}=0
$$

when $z>2.5 \mathrm{~km}$ 
where

$$
A=1.5^{\circ} \mathrm{C}, L=20 d=10 \mathrm{~km} \text { and } D=10 d=5 \mathrm{~km} \text {. }
$$

The lapse rate of mean temperature with height and the mean surface temperature at $t=0$ are assumed in the follwing way;

$$
\begin{aligned}
& \text { case (A): } \frac{\partial \bar{T}}{\partial z}=-1.01^{\circ} \mathrm{C} / 100 \mathrm{~m},(\bar{T})_{z=0}=30^{\circ} \mathrm{C} \\
& \text { case (B): } \frac{\partial \bar{T}}{\partial z}=-1.02^{\circ} \mathrm{C} / 100 \mathrm{~m},(\bar{T})_{z=0}=30^{\circ} \mathrm{C} \\
& \text { case (C): } \frac{\partial \bar{T}}{\partial z}=-1.03^{\circ} \mathrm{C} / 100 \mathrm{~m},(\bar{T})_{z=0}=30^{\circ} \mathrm{C} .
\end{aligned}
$$

Although the relation between the maximum value of kinetic energy and the initial value of $\langle\bar{S}\rangle$ is discussed in (6.10), we consider now the relation between $\langle\bar{K}\rangle_{\max }$ and $\left\langle\bar{w}^{2}\right\rangle_{\max }$. If we assume that

$$
\begin{aligned}
& \left\langle\bar{u}^{2}\right\rangle=\left\langle\left(\overline{\frac{\partial \psi}{\partial z}}\right)^{2}\right\rangle \approx-m^{2} \overline{\left\langle\psi^{2}\right\rangle}, \\
& \left\langle\overline{\left.w^{2}\right\rangle}=\left\langle\left(\overline{\frac{\partial \psi}{\partial z}}\right)^{2}\right\rangle \approx-k^{2} \overline{\left\langle\psi^{2}\right\rangle}\right.
\end{aligned}
$$

where $k$ and $m$ are the characteristic wave numbers in the horizontal and vertical directions respective$1 \mathrm{y}$, the value of $\langle\bar{K}\rangle$ may be expressed by $\left\langle\bar{w}^{2}\right\rangle$ in the form

$$
\langle\bar{K}\rangle \approx \frac{1}{2}\left\langle\bar{w}^{2}\right\rangle\left(1+\frac{m^{2}}{k^{2}}\right) .
$$

In our numerical experiment, of $L=2 D$ we may be able to assume that $k^{2} \approx m^{2}$ and $\langle\bar{K}\rangle \approx\left\langle\bar{w}^{2}\right\rangle$ because the disturbance whose characteristic wave length is $m \approx \pi / D$ will predominate in the vertical direction under the rigid boundaries of $z=0$ and $z=D$. In this situation, the equation (6.10) may be approximated by

$$
\left\langle\bar{w}^{2}\right\rangle_{\max }=-\frac{g z_{M}{ }^{2}}{12}\left[\frac{1}{\left\langle T_{0}\right\rangle}\left(\frac{\partial \bar{T}}{\partial z}+\Gamma\right)\right]_{z=z_{M} / 2, t=0} .
$$

The reason why we try to obtain the relation between $\left\langle\bar{w}^{2}\right\rangle_{\max }$ and $\langle\bar{S}\rangle_{t=0}$ is that the value of $\left\langle\bar{w}^{2}\right\rangle_{\max }$ is closely connected in case of moist convection to the estimation of latent heat released by the condensation.

In Fig. 3, the time evolution of $\left\langle\bar{w}^{2}\right\rangle$ in case (A) is shown. As may be seen in the figure, we have the maximum value of $\left\langle\bar{w}^{2}\right\rangle,\left\langle\bar{w}^{2}\right\rangle_{\max }$ at

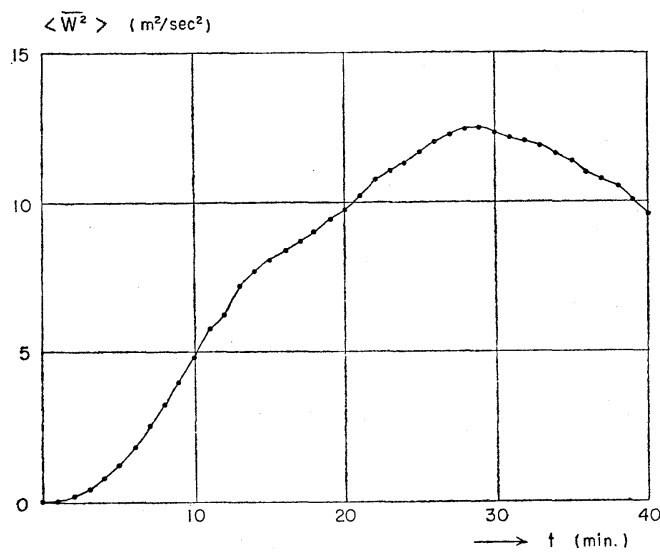

Fig. 3. The evolution of $\left\langle\bar{w}^{2}\right\rangle$ in case of dry convection.

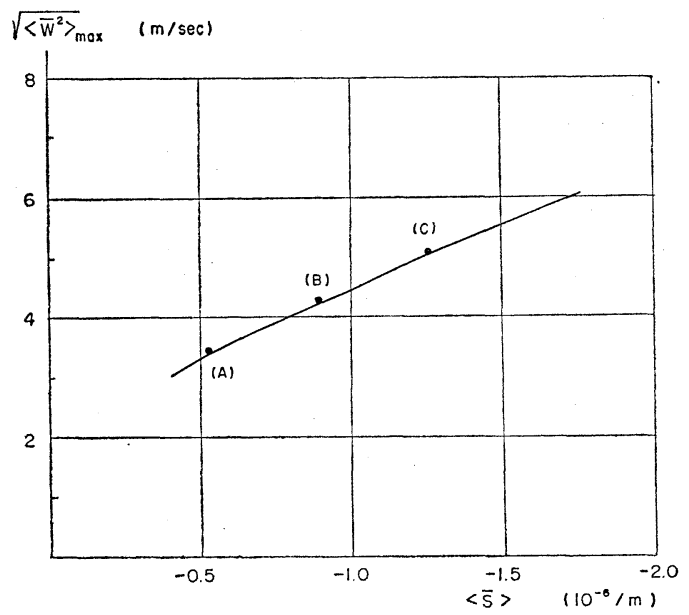

Fig. 4. The values of $\sqrt{\left\langle\bar{w}^{2}\right\rangle_{\max }}$ in cases (A), (B) and (C) are plotted against the value of $\bar{S}$. The full line is the theoretical one.

$t=29 \mathrm{~min}$. The time when we obtain the value of $\left\langle\bar{w}^{2}\right\rangle_{\max }$ depends upon the initial amplitude of $T^{\prime}$ (Gambo and Yoshida). The value of $\left\langle\bar{w}^{2}\right\rangle_{\max }$, however, depends upon the initial stability such as shown in (7.3). In Fig. 4, the relation between $\left\langle\bar{w}^{2}\right\rangle_{\max }$ and

$$
\bar{S} \equiv\left[\frac{1}{\left\langle T_{o}\right\rangle}\left(\frac{\partial \bar{T}}{\partial z}+\Gamma\right)\right]_{z=z_{M} / 2, t=0}
$$

is shown. In the fiugre, the values of $\sqrt{\left\langle\bar{w}^{2}\right\rangle_{\max }}$ in cases (A), (B) and (C) are plotted from the left to right while the theoretical value of $\sqrt{\left\langle\bar{w}^{2}\right\rangle_{\max }}$ estimated by the equation (7.3) is given by the continuous line. As may be seen in the figure, 
there is a good agreement between the result of numerical experiment and the theoretical estimation.

\section{Numerical experiment of moist convection}

The thermodynamic equation is expressed for the moist process in the following way (see Appendix (A. 8) and (A. 9))

$$
\frac{\partial T}{\partial t}+u \frac{\partial T}{\partial x}+w\left(\frac{\partial T}{\partial z}+\Gamma\right)=-w \frac{L}{c_{p}} F^{*}
$$

or

$$
\frac{\partial T}{\partial t}+u \frac{\partial T}{\partial x}+w\left(\frac{\partial T}{\partial z}+\Gamma_{e}\right)=0
$$

where

$$
\begin{aligned}
& \Gamma_{e}=\Gamma-\frac{L}{c_{p}} F^{*} \text { and } F^{*}=\left(\frac{g}{R T}\right) q_{s}\left(\frac{1-\kappa / \lambda}{1+\alpha / \lambda}\right) \\
& \left(\lambda=\frac{R T}{\varepsilon L}, \quad \varepsilon=0.622, \quad \alpha=\frac{L}{c_{p} T} q_{s}, \quad \kappa=\frac{R}{c_{p}},\right. \\
& \left.q_{s}=\text { saturation specific humidity }\right) .
\end{aligned}
$$

Corresponding to the equation (8.1) or (8.2), the continuity equation of moisture may be written in the form (see Appendix (A. 4))

$$
\frac{d q}{d t}=\frac{\partial q}{\partial t}+u \frac{\partial q}{\partial x}+w \frac{\partial q}{\partial z}=0 \quad \text { for } \quad q<q_{s}
$$

$$
\begin{array}{r}
\frac{d q}{d t}=\frac{\partial p}{\partial t}+u \frac{\partial q}{\partial x}+w \frac{\partial q}{\partial z}=w F^{*} \\
\text { for } q \geqq q_{s}
\end{array}
$$

where $q$ is the specific humidity. In case where $q \geqq q_{s}$, the actual computations of $T$ and $q$ are performed in the following way.

(1) Compute the changes of $T$ and $q, \delta T_{o}$ and $\delta q_{o}$, for one time increment, $\Delta t$.

(2) Compute the change of $q_{s}, \delta q_{s}$ corresponding to $\delta T_{o}, i$. e.,

$$
\delta T_{s}=\frac{L}{R_{v} T^{2}} \delta T_{o}
$$

where $R_{v}$ is the gas constant of water vapour.

(3) Compare $\left(q_{s}+\delta q_{s}\right)$ with $\left(q+\delta q_{o}\right)$. If $\left(q_{s}+\right.$ $\left.\delta q_{s}\right) \geqq\left(q+\delta q_{o}\right)$, the computation ends. If $\left(q_{s}+\delta q_{s}\right)<\left(q+\delta q_{o}\right)$, we compute further $\delta T_{1}$ and $\delta q_{1}$ which are given by

$$
\begin{gathered}
\delta q_{1}=\frac{\left(q_{s}+\delta q_{s o}\right)-\left(q+\delta q_{o}\right)}{\left[1+\frac{L}{c_{p}}\left(\frac{\partial q_{s}}{\partial T}\right)\right]} \\
\delta T_{1}=-\frac{L}{c_{p}} \delta q_{1} .
\end{gathered}
$$

The equation (8.6) is a approximately obtained from the following relation

$$
q_{s}\left(T+\delta T_{1}\right)=q+\delta q_{1}
$$

where

$$
\begin{gathered}
q_{s}\left(T+\delta T_{1}\right) \doteqdot q_{s}(T)+\frac{\partial q_{s}}{\partial T} \delta T_{1} \\
=q_{s}(T)-\frac{L}{c_{p}} \frac{\partial q_{s}}{\partial T} \delta q_{1} .
\end{gathered}
$$

The final changes of $T$ and $q$ in this case are summarized in the form of $\delta T_{o}+\delta T_{1}$ and $\delta q_{0}+$ $\delta q_{1}$ respctively.

The numerical experiments in case of moist convection are performed in the following way:

$$
\begin{aligned}
& \text { case (A): } \frac{\partial \bar{T}}{\partial z}=-0.65^{\circ} \mathrm{C} / 100 \mathrm{~m},(\bar{T})_{z=0}=30^{\circ} \mathrm{C} \\
& \text { case (B) }: \frac{\partial \bar{T}}{\partial z}=-0.65^{\circ} \mathrm{C} / 100 \mathrm{~m},(\bar{T})_{z=0}=15^{\circ} \mathrm{C} \\
& \text { case (C) }: \frac{\partial \bar{T}}{\partial z}=-0.65^{\circ} \mathrm{C} / 100 \mathrm{~m},(\bar{T})_{z=0}=5^{\circ} \mathrm{C} .
\end{aligned}
$$

The mean distribution of specific humidity is assumed in all cases as follows:

$$
\begin{aligned}
& \bar{q}=\bar{q}_{s}(\bar{T}) \text { for } z=0 \sim 2.5 \mathrm{~km} \\
& \bar{q}=0.6 \bar{q}_{s}(\bar{T}) \text { for } z=2.5 \sim 5 \mathrm{~km} .
\end{aligned}
$$

The lower half layer is saturated in this case. The initial disturbances superimposed on basic fields mentioned above are

$$
\begin{gathered}
\quad u^{\prime}=0, \quad w^{\prime}=0, \quad q^{\prime}=0 \\
T^{\prime}=A \sin \frac{2 \pi}{L} x \sin \frac{2 \pi}{D} z \quad \text { when } z<2.5 \mathrm{~km} \\
T^{\prime}=0 \quad \text { when } z>2.5 \mathrm{~km}
\end{gathered}
$$

where

$$
A=1.5^{\circ} \mathrm{C}, \quad L=10 \mathrm{~km} \text { and } D=5 \mathrm{~km} .
$$

The domain which we use for the numerical experiment, the grid-length and the time increment $\Delta t$ are the same as those mentioned in case of dry convection, $i$. e.,

$$
x=i \times d, \quad i=1,2, \cdots \cdots I(I=200)
$$




$$
\begin{aligned}
& z=j \times d, \quad j=1,2, \cdots \cdots J(J=11) \\
& d=500 \mathrm{~m}, \quad \Delta t=10 \mathrm{sec} .
\end{aligned}
$$

As the example of numerical experiment, the time evolutions of mean kinetic energy, $\langle\bar{K}\rangle$ in cases (A) and (B) are shown in Fig. 5. On the other hand, the time evolutions of $\left\langle\bar{w}^{2}\right\rangle$ in cases (A) and (B) are also shown in Fig. 6. As may be seen in Figs. 5 and 6 , the maximum value of $\langle\bar{K}\rangle$ in case (A) is about $51 \mathrm{~m}^{2} / \mathrm{sec}^{2}$, while the maximum value of $\left\langle\bar{w}^{2}\right\rangle$ is about $57 \mathrm{~m}^{2} / \mathrm{sec}^{2}$. As mentioned in section 7 we may assume that

$$
\begin{gathered}
\langle\bar{K}\rangle=\frac{1}{2}\left(\left\langle\bar{u}^{2}\right\rangle+\left\langle\bar{w}^{2}\right\rangle\right)=\frac{1}{2}\left\langle\bar{w}^{2}\right\rangle\left(1+\frac{\left\langle\bar{u}^{2}\right\rangle}{\left\langle\bar{w}^{2}\right\rangle}\right) \\
\frac{\left\langle\bar{u}^{2}\right\rangle}{\left\langle\bar{w}^{2}\right\rangle} \approx \frac{k^{2}}{m^{2}}
\end{gathered}
$$

$\langle\bar{K}\rangle\left(\mathrm{m}^{2} / \mathrm{sec}^{2}\right)$

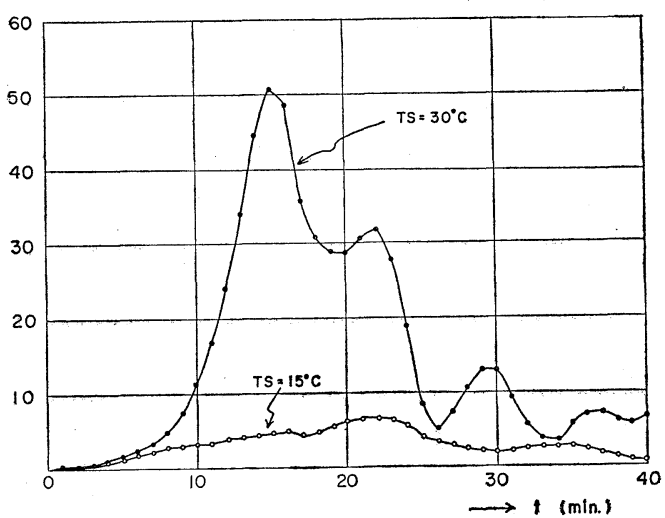

Fig. 5. The evolutions of $\langle\bar{k}\rangle$ in case of moist convection. TS in the figure denotes the value of mean surface temperature.

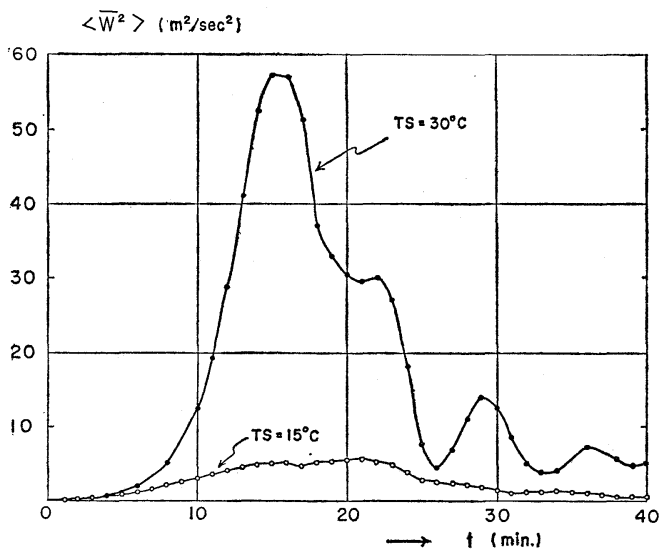

Fig. 6. The evolution of $\left\langle\bar{w}^{2}\right\rangle$. TS in the figure denotes the value of mean surface temperature. where $k$ and $m$ are the characteristic wave numbers in the horizontal and vertical directions respectively.

Since we may put $k^{2} \approx m^{2}$ in our case, because we have $k \approx 2 \pi / L, m \approx \pi / D$ and $L=2 D$, we get

$$
\langle\bar{K}\rangle_{\max } \approx\left\langle\bar{w}^{2}\right\rangle_{\max } .
$$

In this sense, we try to obtain the relation between the decrease of potential energy and the value of $\left\langle\bar{w}^{2}\right\rangle_{\max }$ instead of $\langle\bar{K}\rangle_{\max }$. The energy integral (5.23) in case of shallow convection is expressed by

$$
\begin{aligned}
& \left\langle\overline{\rho_{o} K}\right\rangle-\iint_{1-\sigma} \rho_{o} g z T_{e}^{*} d x d z-\iint_{\sigma} \rho_{o} g z T_{c} * d x d z \\
& =\text { const. }
\end{aligned}
$$

where

$$
\left\langle\overline{\rho_{o} K}\right\rangle=\iint_{1-\sigma} \rho_{o} K d x d z+\iint_{\sigma} \rho_{o} K d x d z .
$$

In case of dry convection, the maximum value of $\langle\bar{K}\rangle$ is obtained assuming that the minimum value of $\langle\bar{P}\rangle$ corresponds to $\langle\bar{K}\rangle_{\max }$. Here the minimum value of $\langle\bar{P}\rangle$ means that the lapse rate of mean temperature, $-\partial \bar{T} / \partial z$ becomes the dry adiabatic lapse rate, $\Gamma$. Considering this result obtained in case of dry convection, we assume the situation of moist convection in such a way that the maximum value of $\langle\bar{K}\rangle$ is obtained when the lapse rate of mean temperature within the region of upward current, $-\partial \bar{T}_{c} / \partial z$ becomes the moist adiabatic lapse rate, $\Gamma_{e}$. When the value of $\left(\frac{\partial \bar{T}_{c}}{\partial z}+\Gamma_{e}\right)$ becomes zero, we may expect some change of the lapse rate of mean temperature within the region of downward current. Since it is difficult problem to discuss quantitatively such situation, we assume as follows:

(a) the cloud amount $\sigma$ is constant with time.

(b) $\left(\iint_{1-\sigma} \rho_{o} g z T_{e}^{*} d x d z\right)$ is constant with time

(c) $\iint_{\sigma} \rho_{o} g z T_{e}^{*} d x d z=\rho_{o}\langle\sigma\rangle g \int z T_{e}^{*} d z$ where $\langle\bar{\sigma}\rangle$ is the mean value of $\sigma$

(d) $\left\langle\overline{\rho_{o} K}\right\rangle=\rho_{o}\langle\bar{K}\rangle=\rho_{o} \frac{1}{2}\left\langle\bar{w}^{2}\right\rangle\left(1+\frac{m^{2}}{k^{2}}\right)$.

The assumption (b) means that the amount of increase or decrease of potential energy within the region of downward current is neglected compared with that in the region of upward 
current. The assumption (d) denotes that $\left\langle\bar{w}^{2}\right\rangle$ $\approx\langle\bar{K}\rangle$ in case of $k^{2} \approx m^{2}$. Here we neglect the change of mean wind field such as $\bar{u}$, under the assumption of $\partial \bar{u} / \partial z=0$ at the initial time. In general, the decrease of potential energy is partly converted to the increase of kinetic energy of mean zonal flow in case where there is the vertical shear of mean zonal flow. However, this problem will not be considered in this paper.

Following these assumptions mentioned above, we may esaily obtain

$$
\langle\bar{K}\rangle_{\max } \approx\left\langle\bar{w}^{2}\right\rangle_{\max }\left(1+\frac{m^{2}}{k^{2}}\right)=\langle\bar{P}\rangle_{t=0}
$$

where $\langle\bar{P}\rangle_{t=0}=-\langle\bar{\sigma}\rangle g\left(\int z \bar{T}_{c}^{*} d z\right) / z_{M}$ and $z_{M}$ is the height of the upper boundary. Since $T_{c}{ }^{\prime}$ is the departure from the wet adiatbat and $T_{c^{\prime}}=0$ at $z=z_{M} / 2$ as difined in (5.12), we have

$$
T_{c}^{\prime}=-\left(\frac{\partial \bar{T}_{c}}{\partial z}+\Gamma_{e}\right)_{z=z_{M} / 2}\left(\frac{z_{M}}{2}-z\right)
$$

where the suffix $c$ denotes the value within the domain of upward current. Thus the value of $\langle\bar{P}\rangle_{t=0}$ may be approximated by

$$
\begin{aligned}
\langle\bar{P}\rangle_{t=0} & =\langle\bar{\sigma}\rangle \frac{g}{z_{M}} \int_{0}^{z_{M}} z\left(\frac{z_{M}}{2}-z\right) \\
& \times\left[\frac{1}{T_{o c}}\left(\frac{\partial \bar{T}_{c}}{\partial z}+\Gamma_{e}\right)\right]_{z=z_{M} / 2, t=0} d z \\
\doteqdot-\langle\sigma\rangle & \frac{g z_{M}{ }^{2}}{12}\left[\frac{1}{T_{o c}}\left(\frac{\partial \bar{T}_{c}}{\partial z}+\Gamma_{e}\right)\right]_{z=z_{M} / 2, t=0} .
\end{aligned}
$$

In our numerical experiment, $k^{2} \approx m^{2}$. Thus from (8.12) and (8.13) we get

$$
\left\langle\bar{w}^{2}\right\rangle_{\max }=-\langle\sigma\rangle \frac{g z_{M}{ }^{2}}{12}\langle S e\rangle_{t=0}
$$

where

$$
\langle\bar{S} e\rangle_{t=0}=\left[\frac{1}{T_{o c}}\left(\frac{\partial \bar{T}_{c}}{\partial z}+\Gamma_{e}\right)\right]_{z=z_{M} / 2, t=0} .
$$

In deriving the relation (8.14), we assume that the depth of conditionally unstable atmosphere is $z_{M}$ which corresponds to the top of boundary. In case where the depth of conditionally unstable atmosphere, $D$ is less than $z_{M}$, the equation may be written in the form

$$
\left\langle\bar{w}^{2}\right\rangle_{\max }=-\langle\bar{\sigma}\rangle^{\frac{g D^{2}}{12}}\left[\frac{1}{T_{o c}}\left(\frac{\partial \bar{T}_{c}}{\partial z}+\Gamma_{e}\right)\right]_{z=D / 2}, t=0 .
$$

In our numerical experiment, the values of $D$ in case (A) is larger than $z_{M}$ and so we may put $D=z_{M}$ in (8.15), while the values of $D$ in cases (B) and (C) are roughly estimated as $4 \mathrm{~km}$ and $2 \mathrm{~km}$, respectively. On the other hand, the values of

$$
-\left[\frac{1}{T_{o c}}\left(\frac{\partial \bar{T}_{c}}{\partial z}+\Gamma_{e}\right)\right]_{z=D / 2}, t=0,
$$

in cases (A), (B) and (C) are estimated as $7.7 \times$ $10^{-6} \mathrm{~m}^{-1}, 3.0 \times 10^{-6} \mathrm{~m}^{-1}$ and $1.0 \times 10^{-6} \mathrm{~m}^{-1}$ respectively. Considering these estimations for $D$ and

$$
\left[\frac{1}{T_{o c}}\left(\frac{\partial \bar{T}_{c}}{\partial z}+\Gamma_{e}\right)\right]_{z=D / 2}, t=0,
$$

the value of $\left\langle\bar{w}^{2}\right\rangle_{\max }$ may be estimated from (8.15) if we have the information about $\langle\bar{\sigma}\rangle$. Similar relation that the maximum vertical velocity of the ascending motion is proportional to $\left(\frac{\partial \bar{T}}{\partial z}+\Gamma_{e}\right)^{1 / 2}$ is also discussed by Kuo (1965) and Asai (1967). In Kuo's case, the proportional constant is obtained as functions of the Rayleigh number and the value of $\sigma$ when the convective motion is stationary.

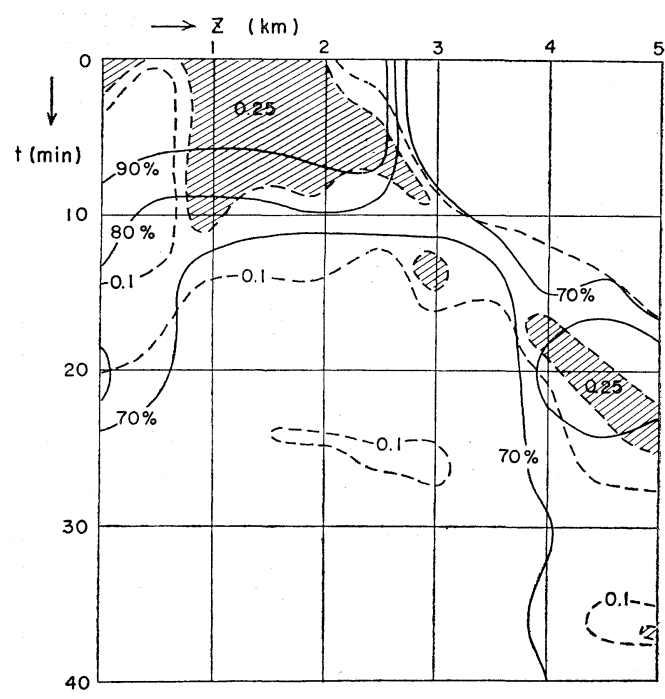

Fig. 7. The evolution of $\bar{\sigma}$ is shown by the dashed line. The shaded part indicates that $\bar{\sigma}>0.25$. The full line corresponds to the relative humidity. 
Since it is very difficult problem to obtain the value of $\sigma$ from the theoretical point of view, we use the empirical result for $\langle\bar{\sigma}\rangle$. In Fig. 7, the time evolution of $\bar{\sigma}$ in case (A) is shown as the function of $z$ by the dashed line. Here $\bar{\sigma}$ is the mean value of $\sigma$ with respect to $x$. The shaded area is for $\bar{\sigma}>0.25$. For the sake of better understanding, the time evolution of relative humidity averaged in the horizontal direction, $q / q_{s}$ is also shown in the figure by the continuous line. As may be seen in the figure, the relative humidity decreases with time, because there is no vapour supply through the boundary of domain in our experiment. Following the decrease of relative humidity with time, the value of $\bar{\sigma}$ tends to zero, starting from $\bar{\sigma}=1 / 2$. In our experiment, the initial value of $\bar{\sigma}$ is assumed as $\bar{\sigma}=1 / 2$ in the lower half layer. Considering this circumstance about $\bar{\sigma}$, the mean value of $\sigma,\langle\bar{\sigma}\rangle$ in (8.14) or (8.15) is assumed as $\langle\bar{\sigma}\rangle=1 / 4$.

In Fig. 8, the relation between the value of $\left\langle\bar{w}^{2}\right\rangle_{\max }$ and the potential energy at the initial time is shown. The ordinate denotes the value of $\sqrt{\left\langle\bar{w}^{2}\right\rangle_{\max }}$ obtained by the numerical experiment, while the abscissa means the value of

$$
\left[-\langle\bar{\sigma}\rangle \frac{g D^{2}}{12}\left\langle\bar{S}_{e}\right\rangle\right]_{t=0}^{1 / 2}
$$

The cases (A), (B) and (C) are marked in the figrue by the symbols (A), (B) and (C) respectively. The continuous line in the figure shows the

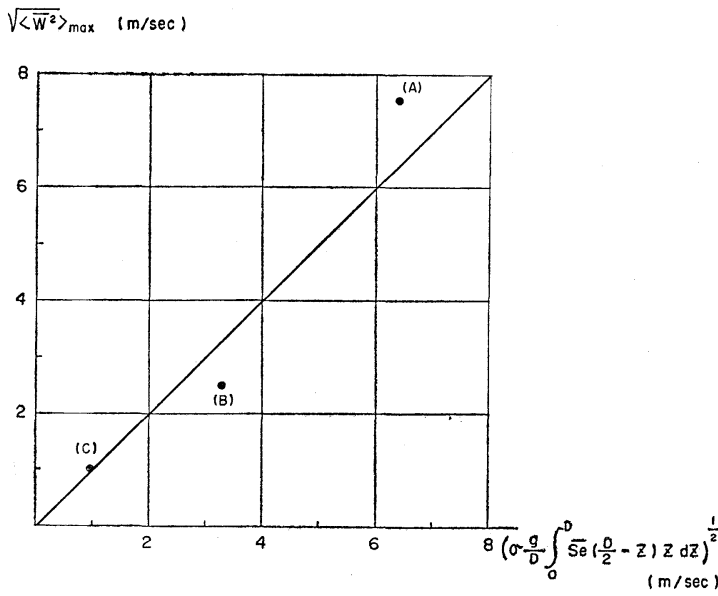

Fig. 8. The values of $\sqrt{\left\langle\bar{w}^{2}\right\rangle_{\max }}$ in cases (A), (B) and (C) are plotted against the value of $\bar{S} e$. The full line is the theoretical one. relation expressed by (8.15) where $\langle\bar{\sigma}=1 / 4$. As may be seen in the figure, the assumptions (a)(d) mentioned before may be regarded as reasonable ones as the sense of first approximation. If we want to obtain the higher approximation for $\left\langle\bar{w}^{2}\right\rangle_{\max }$, the assumption (b) must be revised, taking into account the time change of $T_{e}^{*}$ due to $\left\langle\bar{w}^{2}\right\rangle_{\max }$ which is obtained by the first approximation.

\section{Cloud amount and precipitation}

In the previous section, we pick up the case where the lower half layer is saturated at the initial time. So, the cloud amount $\sigma$ starts from $\bar{\sigma}=1 / 2$ in the lower half layer. On the other hand, the mean value of $\sigma$ in case of shallow convection is regarded as the order of $1 / 100$. Kasahara and Asai (1967) also obtained in the numerical experiment that when the moist active cloud system developed, the value of $\sigma$ was several percent. In order to consider this problem, the following numerical experiment is performed.

The mean distributions of temperature and specific humidity are as follows:

$$
\begin{array}{cl}
\frac{\partial \bar{T}}{\partial z}=-0.65^{\circ} \mathrm{C} / 100 \mathrm{~m}, & (\bar{T})_{z=0}=30^{\circ} \mathrm{C} \\
\bar{q}=0.8 \bar{q}_{s}(\bar{T}) & \text { for } \quad z=0 \sim 2.5 \mathrm{~km} \\
\bar{q}=0.6 \bar{q}_{s}(\bar{T}) & \text { for } \quad z=2.5 \sim 5.0 \mathrm{~km} .
\end{array}
$$

The initial disturbances superimposed on basic fields mentioned above are

$$
\begin{array}{ll}
\qquad u^{\prime}=0, \quad w^{\prime}=0, \quad q^{\prime}=0 \\
T^{\prime}=A \sin \frac{2 \pi}{L} x \sin \frac{2 \pi}{D} z & \text { when } z<2.5 \mathrm{~km} \\
T^{\prime}=0 & \text { when } z>2.5 \mathrm{~km} \\
\qquad A=1.5^{\circ} \mathrm{C}, \quad L=10 \mathrm{~km} \quad \text { and } \quad D=5 \mathrm{~km} .
\end{array}
$$

where

This case corresponds to case (A) in section 8 except the distribution of mean specific humidity in the lower half layer. In case (A), the lower half layer is saturated at the initial time while the air is not saturated in this case. The time evolution of $\bar{\sigma}$ is shown in Fig. 9 as the function of $z$. Comparing with Fig. 7, the remarkable difference is that the condensation occurs after 7 min. from the initial time and the cloud base is about $1 \mathrm{~km}$ in this case. At about $t=30 \mathrm{~min}$, the cloud top approached at the top of boundary. 


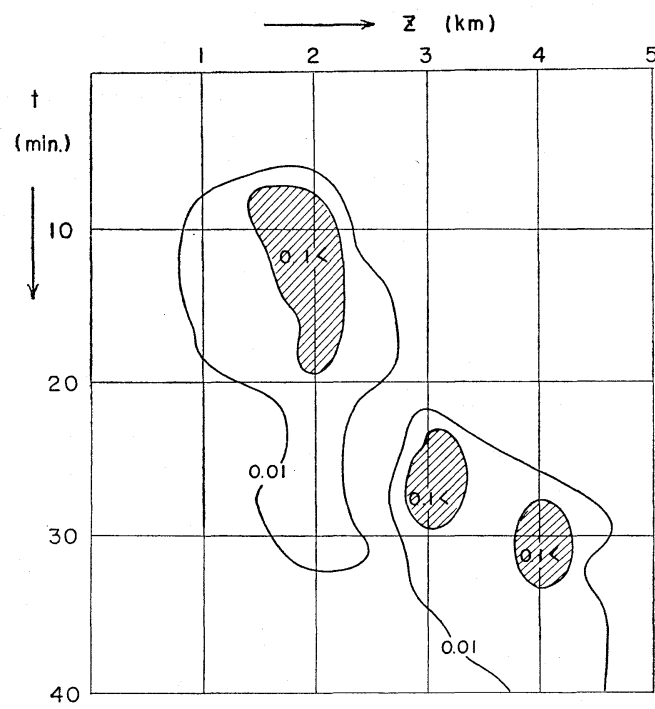

Fig. 9. The evolution of $\bar{\sigma}$. The shaded part indicates that $\bar{\sigma}>0.1$

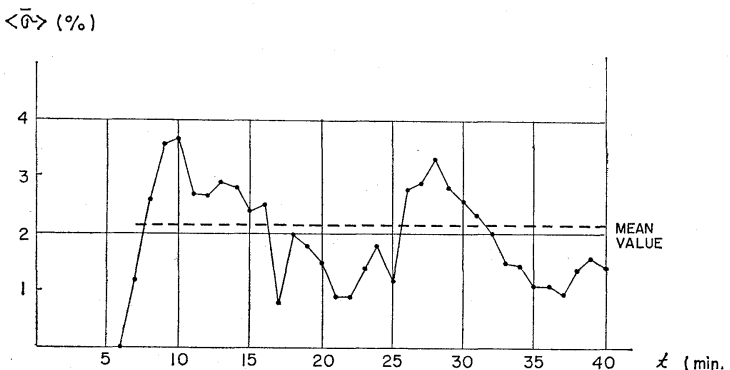

Fig. 10. The evloution of $\langle\bar{\sigma}\rangle$ in unit of $\%$. The mean value of $\langle\bar{\sigma}\rangle$ during the time interval of $t=6 \sim 40^{\circ} \mathrm{min}$. is denoted by the dashed line.

$\sqrt{\left\langle\bar{W}^{2}\right\rangle}(\mathrm{m} / \mathrm{sec})$

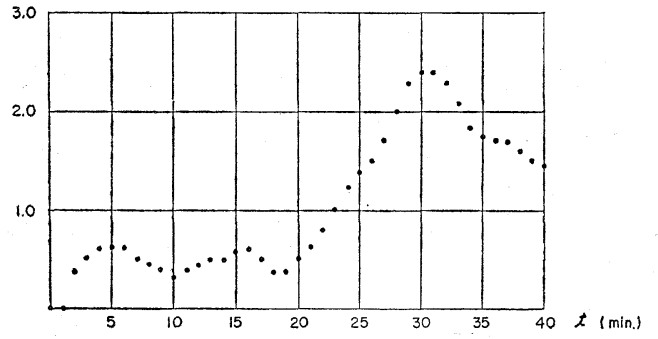

Fig. 11. The evolution of $\sqrt{\left\langle\bar{w}^{2}\right\rangle}$.

In Fig. 10, the time change of $\langle\bar{\sigma}\rangle$ is shown. The mean value of $\langle\sigma\rangle$ during the time interval of $t=7 \sim 40 \mathrm{~min}$. is about 0.021 . If we consider the equation (8.15), the value of $\sqrt{\left\langle\bar{w}^{2}\right\rangle_{\max }}$ may be expected as $1.9 \mathrm{~m} / \mathrm{sec}$, because

$$
-\left[\frac{1}{T_{o c}}\left(\frac{\partial \bar{T}_{c}}{\partial z}+\Gamma_{e}\right)\right]_{z=D / 2}, t=0
$$

is $7.7 \times 10^{-6} \mathrm{~m}^{-1}$ as mentioned in the previous section. In Fig. 11, the time change of $\sqrt{\left\langle w^{2}\right\rangle}$ is also shown and we may find the approximate value of $\sqrt{\left\langle\bar{w}^{2}\right\rangle_{\max }}$ mentioned above.

Now we consider the problem of precipitation. The amount of precipitation, Prec. for the entire column is computed from

$$
\text { Prec. }=-\int_{0}^{z_{M}} \rho \delta q_{s} d z
$$

where $\delta q_{s}=\frac{d q_{s}}{d t} \Delta t$ and $\Delta t$ is the time unit.

Since $\frac{d q_{s}}{d t}=-w_{c} F^{*}$ as explained in the Appendix, we have

$$
\text { Prec. } \div\langle\rho\rangle \Delta t \int_{0}^{z_{M}} w_{c} F^{*} d z
$$

where $w_{c}$ is the upward current.

Since $F^{*}$ is the function of $p, \bar{T}$ and $\bar{q}_{s}$, the important factor for estimating the amount of precipitation is to obtain the value of $w_{c}$. If we assume that the measure of cloud area $\sigma$ is constant, we may have

$$
\begin{gathered}
\langle\bar{w}\rangle \equiv \sigma\left\langle\bar{w}_{c}\right\rangle+(1-\sigma)\left\langle\bar{w}_{e}\right\rangle=0, \\
\left(\bar{w}_{c} \equiv \int_{\sigma} w_{c} d x, \bar{w}_{e} \equiv \int_{1-\sigma} w_{e} d x\right) \\
\left\langle\bar{w}_{e}\right\rangle=-\left(\frac{\sigma}{1-\sigma}\right)\left\langle\bar{w}_{c}\right\rangle
\end{gathered}
$$

where $w_{e}$ is the downward current.

From the definition of $\left\langle\bar{w}^{2}\right\rangle$, we get

$$
\left\langle\bar{w}^{2}\right\rangle=\sigma\left\langle\bar{w}_{c}^{2}\right\rangle+(1-\sigma)\left\langle\bar{w}_{e}^{2}\right\rangle .
$$

Assuming that $\left\langle\bar{w}_{e}^{2}\right\rangle=\left\langle\bar{w}_{e}\right\rangle^{2}$, we have from (9.3) and (9.4) the following relation

$$
\left\langle\bar{w}^{2}\right\rangle=\left(\frac{\sigma}{1-\sigma}\right)\left\langle\bar{w}_{c}^{2}\right\rangle .
$$

If the equation (9.1) is approximated by

$$
\text { Prec. } \div\langle\bar{\rho}\rangle \Delta t \sqrt{\left\langle\bar{w}_{c}^{2}\right\rangle} \int_{0}^{z_{M}} F^{*} d z
$$




$$
\text { Prec. }=\langle\rho\rangle \Delta t\left(\frac{1-\sigma}{\sigma}\right)\left\langle\bar{w}^{2}\right\rangle \int_{0}^{z_{M}} F^{*} d z,
$$

the amount of precipitaion is easily obtained as the function of $\sigma$ and $\left\langle\bar{w}^{2}\right\rangle$ which is closely connected with the mean stability.

In closing this section, we summarize the general principle where we represent the characteristic feature of moist shallow convection. The vertical velocity in case of shallow convection is approximately obtained as the function of the cloud amount, $\sigma$ and the mean stability of the atmosphere, $\left(\frac{\partial \bar{T}}{\partial z}+\Gamma_{e}\right)$ in the vertical direction. This result comes from the assumption that the lapse rate of mean temperature within the region of upward current becomes the moist adiabatic lapse rate, if convective motion occurs.

In this paper, however, we consider the isolated system where there is no supply of water vapour or sensible heat through the boundary of domain we concerned with. In this sense, the convective motion does not continue for a long time as may be seen in Fig. 7 or Fig. 9. The supply of water vapour or sensible heat through the lower boundary will change the situation mentioned above. In connection with this problem, the shear of mean horizontal wind velocity with height also plays an important role. These effects will be discussed in the coming paper as the problem of interaction between the motion of large-scale disturbances and the small-scale convection.

\section{Acknowledgements}

The authors wish to experess their hearty thanks to the staff members of our Computaiton Center for valuable discussions. Thanks are due to Misses K. Endo and Y. Togo for typing the draft and drawing the figures.

\section{References}

Asai, T., 1967: On the characteristics of cellular cumulus convection. J. meteor. Soc. Japan, 45, 251-260.

Gambo, K. and T. Yoshida, 1967: The characteristic feature of thermal convection in the atmosphere (I) - case of dry convection-. J. meteor. Soc. Japan. 45, 111-125.

Kasahara, A and T. Asai, 1967: Effects of an ensemble of convective elements on the large-scale motions of the atmosphere. J. meteor. Soc. Japan. 45, 280291.

Kuo, H.L., 1965: Further studies of the properties of cellular convection in a conditionally unstable atmosphere. Tellus, 17, 4, 413-433.

Leith, C. E., 1965: Convection in a six-level model atmosphere. Proc. Int. Symposium of Dynamics of Large-Scale Atmospheric Processes, 134-138.

Ogura, Y. and N.A. Phillips, 1962: Scale analysis of deep and shallow convection in the atmosphere. J. atmos. Sci., 19, 173-179.

\section{Appendix}

Let $q_{s}$ be the saturation specific humidity. The equation for $\frac{d}{d t} q_{s}$ is

$$
\frac{d q_{s}}{d t}=\frac{\partial q_{s}}{\partial t}+u \frac{\partial q_{s}}{\partial x}+w \frac{\partial q_{s}}{\partial z}
$$

Since $q_{s}=q_{s}(p, T)$, the equation (A.1) may be approximated by

$$
\begin{aligned}
\frac{d q_{s}}{d t}= & \left(\frac{\partial T}{\partial t}+u \frac{\partial T}{\partial x}\right)\left(\frac{\partial q_{s}}{\partial T}\right)_{p}+\left(\frac{\partial p}{\partial t}+u \frac{\partial p}{\partial x}\right) \\
& \times\left(\frac{\partial q_{s}}{\partial p}\right)_{T}+w \frac{\partial q_{s}}{\partial z} \approx\left(\frac{\partial T}{\partial t}+u \frac{\partial T}{\partial x}\right) \\
& \times\left(\frac{\partial q_{s}}{\partial T}\right)_{z}+w \frac{\partial q_{s}}{\partial z}
\end{aligned}
$$

where the subscirpts $p, T$ and $z$ denote the differentiations on the surfaces of $p=$ const., $T=$ const. and $z=$ const. respectively.

Here we have assumed that

$$
\left|\frac{\partial q_{s}}{\partial T} \delta T\right| \gg\left|\frac{\partial q_{s}}{\partial p} \delta p\right| .
$$

On the otherhand, we have from the thermodynamic equation, assuming that

$$
\frac{d Q}{d t}=-\frac{L}{c_{p}} \frac{d q_{s}}{d t},
$$

the following equation for $T$

$$
\frac{\partial T}{\partial t}+u \frac{\partial T}{\partial x}+w\left(\frac{\partial T}{\partial z}+\Gamma\right)=-\frac{L}{c_{p}} \frac{d q_{s}}{d t}
$$

where $\Gamma=g / c_{p}$ and $L$ is the latent heat of condensation. Replacing (A.2) into the right-hand side of (A.3), we get

$$
\frac{\partial T}{\partial t}+u \frac{\partial T}{\partial x}=\frac{-w\left[\left(\frac{\partial T}{\partial z}+\Gamma\right)+\frac{L}{c_{p}} \frac{\partial q_{s}}{\partial z}\right]}{\left(1+\frac{L}{c_{p}} \frac{\partial q_{s}}{\partial T}\right)}
$$


Thus the final form for $d q_{s} / d t$ becomes as follows where

$$
\frac{d q_{s}}{d t}=w F^{*}
$$

where

$$
\begin{aligned}
F^{*} & =\frac{\left[\frac{\partial q_{s}}{\partial z}-\left(\frac{\partial q_{s}}{\partial T}\right)_{z}\left(\frac{\partial T}{\partial z}+\Gamma\right)\right]}{\left[1+\frac{L}{c_{p}}\left(\frac{\partial q_{s}}{\partial T}\right)_{z}\right]} \\
& =\frac{\left[\left(\frac{\partial q_{s}}{\partial z}\right)_{T}-\Gamma\left(\frac{\partial q_{s}}{\partial T}\right)_{z}\right]}{\left[1+\frac{L}{c_{p}}\left(\frac{\partial q_{s}}{\partial T}\right)_{z}\right]} .
\end{aligned}
$$

In order to simplify the equation (A.5), we use the following relations

$$
\begin{aligned}
& \left(\frac{\partial q_{s}}{\partial T}\right)_{z} \div\left(\frac{\varepsilon L}{R T}\right) \frac{q_{s}}{T} \quad(\varepsilon=0.622) \\
& \left(\frac{\partial q_{s}}{\partial z}\right) \div \frac{\partial q_{s}}{\partial p} \frac{\partial p}{\partial z}=\frac{g}{R T} q_{s} .
\end{aligned}
$$

Here we assume that $\frac{\partial q_{s}}{\partial p}=-\frac{q_{s}}{p}$. The replacement of these equations in (A.5) gives

$$
F^{*}=\left(\frac{g}{R T}\right) q_{s}\left(\frac{1-\kappa / \lambda}{1+\alpha / \lambda}\right)
$$

$$
\lambda=\frac{R T}{\varepsilon L}, \quad \alpha=\frac{L}{c_{p} T} q_{s} \text { and } \kappa=\frac{R}{c_{p}} .
$$

From (A.3), (A.4) and (A.6), we may easily obtain the rate of change of temperature in the following way:

(a) case of adiabatic motion:

$$
\frac{\partial T}{\partial t}+u \frac{\partial T}{\partial x}+w\left(\frac{\partial T}{\partial z}+\Gamma\right)=0
$$

(b) case of non-adiabatic motion:

$$
\frac{\partial T}{\partial t}+u \frac{\partial T}{\partial x}+w\left(\frac{\partial T}{\partial z}+\Gamma\right)=-w \frac{L}{c p} F^{*}
$$

or

$$
\frac{\partial T}{\partial t}+u \frac{\partial T}{\partial x}+w\left(\frac{\partial T}{\partial z}+\Gamma_{e}\right)=0
$$

where $\Gamma_{e}$ is the moist adiabatic lapse rate of temperature and is given by

$$
\Gamma_{e}=\Gamma+\frac{L}{c_{p}} F^{*}=\Gamma\left(\frac{1+\alpha / \kappa}{1+\alpha / \lambda}\right)
$$

\section{大気中における対流の特性について（II ) \\ 一湿潤大気中の対流— \\ 岸保勘三郎・町田英三郎 \\ （気象庁電子計算室）}

じょら乱のスケール解析といら立場から, 湿潤大気中の対流を取扱い, 浅い対流中に括けるェネルギー保存則（運 動エネルギーとポテンシャルエネルギー) を求めた。

このようにして求めたェネルギー保存則より, 対流中の最高上昇速度を, 雲量および大規模スケールでの安定度の パラメーターとして求めた。

上述の定量的議論を裏付けるために, 湿潤大気中の対流に関する数值実験を行なってみた。結果は大体満足でさる ものであった。 